\title{
Que vaut encore la médecine ambulatoire?
}

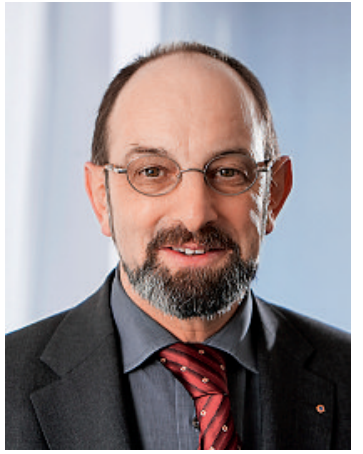

Ernst Gähler
En tant que médecin de famille et médecin de premier recours d'une région rurale, j'ai de plus en plus l'impression d'appartenir à une espèce en voie de disparition. Le renforcement de la médecine de famille est dans toutes les bouches, mais j'ai du mal à croire à la mise en pratique de cette volonté. Mon activité est considérée comme essentielle pour les soins de santé de notre pays. Mon travail est économique et de bonne qualité, je suis toujours atteignable et à la disposition de mes patients. Ces derniers apprécient mon travail et les prestations que je leur fournis depuis 25 ans. J'aurais encore beaucoup de plaisir à exercer mon activité si ce n'étaient les bâtons que les organismes payeurs et les offices fédéraux ne cessent de nous mettre dans les roues.

\section{Les jeunes médecins de famille doivent bénéficier à l'avenir de conditions qui rendent attrayant leur métier de praticien}

Espérons que l'histoire de la médecine de famille suisse ne poursuivra pas dans cette voie et que la collègue qui me succèdera aura encore du plaisir à exercer sa profession. Pour cela, des signes clairs de l'Office fédéral de la santé publique (OFSP) et du monde politique sont nécessaires. Je m'engage pour que les jeunes médecins de famille bénéficient encore à l'avenir de conditions rendant attrayant leur métier de praticien indépendant; le laboratoire, la dispensation directe de médicaments, la radiographie, l'ECG, etc. contribuent notamment à l'intérêt de notre tâche.

Pour que nous puissions continuer à exercer en tant que médecins de famille, la mise en œuvre d'idées et de solutions créatives et constructives s'impose. Mais il est fréquent que de telles idées soient tout simplement étouffées dans l'œuf, sous le couvert de pseudo-mesures d'économie de la Confédération. Ainsi, les responsables concernés n'ont rien voulu savoir du tarif «Point-of-Care», notre proposition constructive pour le laboratoire du praticien. Les calculs de l'OFSP se basent, selon nous, sur des données incompréhensibles qui s'avèrent arbitraires. Nous n'avons pas encore abandonné la lutte pour le laboratoire de cabinet médical, même si le tarif du conseiller fédéral Couchepin a de grandes chances d'être introduit le $1^{\text {er }}$ juillet 2009.
Les médecins en cabinet sont maintenant confrontés à de nouveaux déboires. A la demande du conseiller fédéral Couchepin, le Conseil fédéral a décidé des mesures d'urgence visant à maîtriser les coûts, qui vont être soumises au Parlement. Ces mesures ne convainquent pas, d'une part parce qu'elles ne contribueront pas à la maîtrise des coûts, et d'autre part parce qu'elles touchent encore une fois les mauvaises personnes, à savoir les patients et les médecins praticiens. Pourtant, la pratique ambulatoire privée est une branche de la santé qui ne représente qu'un quart des dépenses de santé et dont l'augmentation des coûts de 2,5\% depuis 1999 est nettement inférieure à la moyenne suisse de 4,5\%. La neutralité des coûts et la convention prestations-prix (CPP) ont été respectées et les valeurs respectives du point tarifaire adaptées aux différentes modifications. Or, il est maintenant prévu d'intervenir une nouvelle fois à ce niveau ...

Et pourtant, c'est déjà bien assez

- que dans le domaine AA/AI/AM, les exigences d'adaptations de la valeur du point tarifaire, justifiées et reconnues par les assureurs, ne cessent d'être retardées par des changements permanents des règles du jeu, alors que ces modifications correspondent aux conventions contractuelles;

- que dans le domaine LAMal, malgré la CPP, santésuisse informe dans un courrier du 28 avril 2009 qu'aucune adaptation ne sera effectuée et

- que les propositions constructives du corps médical concernant la révision de la Liste des analyses au cabinet médical aient été étouffées sans discussion avec des arguments complètement creux.

\section{Les mesures d'urgence ne contri-}

\section{bueront pas à la maîtrise des coûts}

Se refusant à tolérer ces injustices, la base du corps médical s'insurge et l'appel à la désobéissance se fait plus fort.

Et maintenant? La FMH, la CCM, la SSMG, le CMPR et d'autres sociétés de discipline travaillent de manière intensive au développement et à la négociation de propositions constructives, proactives et orientées vers les solutions. Mais nous réfléchissons également à des scénarios alternatifs, moins constructifs. Nous allons augmenter la pression. Nous le devons à notre base, à nos successeurs et aux patients. Tous souhaitent en effet que nous conservions une médecine de famille et de cabinet économique et de bonne qualité.

Dr Ernst Gähler, vice-président de la FMH, responsable du domaine Tarifs et conventions 